\title{
Experimental characterisation of a cohesive zone model using digital image correlation
}

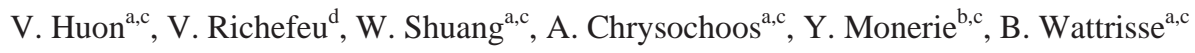 \\ ${ }^{a}$ LMGC, CNRS - Université Montpellier 2, CC 048, Place E. Bataillon, F-34095 Montpellier, \\ France \\ ${ }^{\mathrm{b}}$ IRSN, bat. 702, CE Cadarache, BP3-13115 Saint-Paul-lez-Durance Cedex, France \\ ${ }^{\mathrm{c}}$ MIST Laboratory, IRSN-CNRS-UM2, France \\ ${ }^{\mathrm{d}}$ L3S, Université Grenoble, France
}

\section{Topic}

In recent years, cohesive-zone models have been formulated and used to numerically simulate the fracture of solid materials. Cohesive-zone models presented in the literature involve a 'jump' in the displacement field describing crack onset within a predefined interface network corresponding to interfaces between elements of the finite element (FE) mesh. The introduction of a virtual displacement jump is convenient to numerically manage micro-crack or void initiation, growth and coalescence. Until now, the forms of interface laws were mainly chosen in connection with the overall responses of specimens when subjected to standard loadings. In this study, a cohesive-zone model identification method is proposed based on the local material behaviour derived from kinematical measurements obtained by digital image correlation (DIC). A series of tensile loadings were performed for several damageable elastic-plastic materials on standard tensile specimens. Kinematical data analysis enabled early detection and tracking of the zone where the crack occurs. The results of this study highlight the potential of DIC to quantify damage and show how damage assessments can be inserted in cohesive-zone model identification.

\section{Rough outline}

Cohesive-zone models (CZMs), which were first introduced through the pioneering work of Dugdale [1] and Barenblatt [2], are suitable for simulating fracture in a wide range of materials and accounting for heterogeneities at various scales from the grain up to the structure. In the cohesive/volumetric finite element framework, CZMs are introduced at interfaces between adjacent elements of a finite element discretization. They have been successfully used to simulate and predict the entire fracture process from crack onset to rupture (including crack growth, propagation, potential bifurcation, multiple fracturing, etc.). In recent years, substantial progress has been achieved from a numerical standpoint and CZM predictions have been regularly improved. Investigations were performed on the influence of the shape of CZMs (e.g. bilinear, exponential, and trapezoidal) [3-5].

In this paper, we outline a simple, pragmatic method to identify the normal part of the cohesive law (1D approach). The identification involves strain field measurements during tensile loadings on standard specimens. The image analysis allows early detection and tracking of the material zone 
where the strain localization occurs. This zone is characterized by an inflection point of the longitudinal velocity profile. The 1D approach can be warranted by the kinematical data which shows very low elastoplastic rotations, even in the localization zone. This zone is, as expected, the region where necking occurs and damage preferentially develops.

The measurements of strains $\varepsilon_{1}$ and $\varepsilon_{2}$ and load $f$ enabled us to estimate fields of isotropic damage $D=\alpha_{\mathrm{D}}\left(e^{\varepsilon_{1}+2 \varepsilon_{2}}-1\right)^{2 / 3}$ and, under the transverse isotropy hypothesis, the apparent axial stress $\sigma=e^{-2 \varepsilon_{2}} f / S_{0}, S_{0}$ being the initial cross section, the effective stress $\sigma_{\text {eff }}=\sigma /(1-D)$. The material behaviour is split into an incompressible elastic plastic bulk behaviour and a cohesive surface behaviour that sums up the damage effects. The axial strain $\varepsilon_{1}$ is the sum of a bulk strain $\varepsilon_{\mathrm{B}}$ and a cohesive strain $\varepsilon_{\mathrm{C}}$. Finally, the incompressible stress associated with the bulk behaviour is defined by $\sigma_{\text {inc }}=e^{\varepsilon_{1}} f / S_{0}$.

Figure 1 shows the apparent stress $\sigma$ in the localization zone as a function of the overall and cohesive zone strains. The stress-strain relation $\sigma_{\text {inc }} v s . \varepsilon_{\mathrm{B}}$ associated with the virtual isochoric straining is also plotted on the same graphs. This relation corresponds to the bulk behaviour that should be resolved at the Gauss points in a FE method for each material, and therefore it should be used for determining the bulk parameters of constitutive equations. The relation $\sigma v s . \varepsilon_{\mathrm{C}}$ provides access to the cohesive zone response and corresponds to the CZM, which can be directly used in CZM-based simulations through incorporation of a characteristic length $l_{f e}$ corresponding to the link between the displacement jump $[\mathrm{u}]$ and the cohesive strain $\varepsilon_{\mathrm{C}}$.

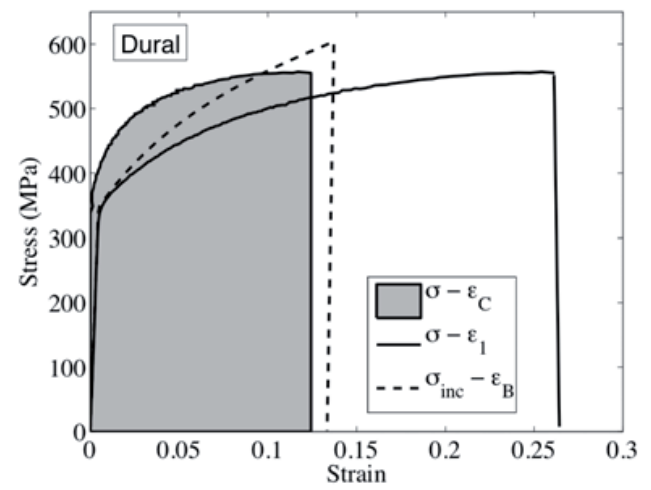

Figure 1: Local (within the localization zone) stress-strain diagrams for the studied material (dural). Measured $\sigma$ vs. $\varepsilon_{11}$ relation (solid line); Identified $\sigma_{\text {inc }} v s . \varepsilon_{\mathrm{C}}$ relation (dashed line); Identified cohesive zone response (solid line with grey surface)

\section{References}

1. D. S. Dugdale, J. of the Mech. and Phys. of Sol., 8, 100-104, (1960)

2. G. I. Barenblatt, Adv. Appl. Mech., 7, 55-129, (1962)

3. N. Chandra, H. Li, C. Shet, H. Ghonem, Int. J. of Sol. and Struct., 39, 2827-2855, (2002)

4. G. Alfano, Composites Sc. and Techn., 66, 723-730, (2006)

5. V. Tvergaard, J. W. Hutchinson, J. Mech. Phys. Sol., 40, 1377-1397, (1992) 